\title{
Leucemia Cutis
}

\author{
Francisco Javier Saldívar Alarcón ${ }^{\mathrm{a}}$, José Luis del Burgo Fernández
}

${ }^{\text {a }}$ Residente de $4^{\circ}$ año de Medicina Familiar y Comunitaria. Centro de Salud de Porzuna (Ciudad Real).

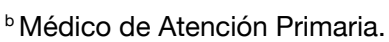
Tutor de residentes. Centro de Salud de Porzuna (Ciudad Real).

Correspondencia: Francisco Javier Saldívar Alarcón, Centro de Salud de Porzuna, 13120 Porzuna, Ciudad Real, España. Correo electrónico: pacsal@gmail.com

Recibido el 2 de febrero de 2012.

Aceptado para su publicación el 1 de sepiembre de 2012.

\section{RESUMEN}

La Leucemia Cutis (LC) es una enfermedad poco frecuente y se asocia con leucemia mieloide aguda la mayoría de las veces. Puede manifestarse antes o después del diagnóstico de leucemia en sangre o médula ósea. No tiene lesiones patognomónicas con respecto al tipo de leucemia con la que se acompaña y el tipo de las lesiones pueden orientar el diagnóstico hacia otro tipo de patologías antes de pensar en LC. Esta enfermedad cursa con un pobre pronóstico, dado que tiene un periodo de sobrevida muy bajo desde el momento de su diagnóstico, a pesar de que con el tratamiento suelen desaparecer los signos clínicos.

Palabras clave: Leucemia, Leucemia mieloide aguda, Leucemia mielomonocítica crónica

\section{ABSTRACT}

Leukaemia Curtis (LC) is a rare disease and is associated with acute myelitis leukaemia most of the time. It can occur before or after diagnosis of leukaemia in blood or bone marrow. It does not have pathogenic lesions compared to the type of leukaemia it is accompanied by and the type of lesions can point diagnosis towards other kinds of pathologies before considering LC. This disease has a poor prognosis, given that it has a very low survival period from the time of diagnosis, even though clinical signs disappear with treatment.

Key words: Leukemia, Leukemia myeloid acute, Leukemia myelomonocytic chronic.

\section{INTRODUCCIÓN}

La leucemia es una neoplasia maligna del sistema hematopoyético que tiene manifestaciones extramedulares en algunos órganos del cuerpo, como la piel $^{1}$. En el $25-40 \%$ de los pacientes con linfomas y leucemias se presentan manifestaciones cutáneas que se clasifican en específicas o inespecíficas ${ }^{2}$.

Las manifestaciones cutáneas inespecíficas, también denominadas leucemides, son observadas con mayor frecuencia y son un resultado anormal de la hematopoyesis o como expresión de desórdenes paraneoplásicos cutáneos. Los signos inespecíficos más comúnmente observados son púrpura, petequias y equimosis, prurito generalizado, eritrodermia exfoliativa, herpes zóster, úlceras crónicas secundarias a herpes simple, pioderma gangrenoso, eritema multiforme, urticaria, hiperpigmentación, micosis cutáneas y erupciones morbiliformes no específicas ${ }^{1,2}$.

Las manifestaciones cutáneas específicas, conocidas como Leucemia Cutis (LC) incluyen todas las lesiones caracterizadas por el infiltrado de células leucémicas, independientemente de su morfología clínica, y pueden corresponder a pápulas, nódulos y placas discretas, de consistencia firme, purpuráceas o de color rojizo-marrón o de aspecto hemorrágico. Las placas leucémicas pueden causar engrosamiento de piel cabelluda, cejas y mejillas produciendo la típica facies leonina ${ }^{1,2}$.

El infiltrado cutáneo por leucemia mielomonocítica crónica (LMMC) es poco frecuente y se considera como signo de progresión de la enfermedad o de transformación a forma aguda ${ }^{3}$. 


\section{OBSERVACIONES CLÍNICAS}

Se trata de un paciente de 85 años de edad, alérgico a doxiciclina y nistatina, que consulta por lesiones cutáneas dolorosas, principalmente las de localización craneal, y de varios días de evolución. Como antecedentes personales, padece hipertensión arterial esencial, diabetes mellitus insulinodependiente, insuficiencia renal crónica, obesidad, vitiligo generalizado, enfermedad pulmonar obstructiva crónica, hipertrofia benigna de próstata, hiperuricemia, ansiedad, espondiloartrosis y leucemia mielomonocítica crónica (LMMC) diagnosticada 7 meses antes.

Sigue tratamiento con dutasterida, palma enana, tamsulosina, insulina mixta, ácido acetilsalicílico, bromuro de ipratropio, salmeterol/fluticasona, teofilina, salbutamol, ácido fólico, alopurinol, losartán, amlodipino, pantoprazol, mirtazapina, megestrol, tramadol, lactulosa e hidroxiurea (que el paciente había abandonado en las semanas previas).

A la exploración física encontramos una tensión arterial de 151/63 mmHg, una frecuencia cardiaca de 83 latidos por minuto y una temperatura de $36,6^{\circ} \mathrm{C}$. Presenta abundantes lesiones cutáneas de aspecto homogéneo diseminadas en tronco, extremidades, cara y cuero cabelludo (figuras 1 y 2 ). Se trata de placas redondeadas de tamaños entre 5 y $10 \mathrm{~mm}$ de diámetro, de coloración eritematoviolácea, no descamativas, de aspecto edematoso e infiltradas al tacto (figura 3). No presenta lesiones en la mucosa oral, palmas ni plantas. En la exploración abdominal no hay masas ni megalias palpables. Las lesiones llevan 2 semanas de evolución, refiriendo que algunas de las que se asientan sobre planos óseos son dolorosas.

El paciente fue derivado a atención hospitalaria por sospecha de LC, presentando la siguiente analítica: Hemograma: leucocitos $11.900 / \mu \mathrm{l}$, segmentados $4.400 / \mu \mathrm{l}$, linfocitos $2.300 / \mu \mathrm{l}$, monocitos $5.100 / \mu \mathrm{l}$, eosinófilos $0 / \mu \mathrm{l}$, hematíes $4.31 \times 10^{6} / \mu \mathrm{l}$, hemoglobina $9,1 \mathrm{~g} / \mathrm{dl}$, hematocrito $28,8 \%$, VCM 66,9 fl, CHCM

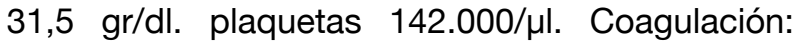
Normal. Bioquímica: Glucosa $130 \mathrm{mg} / \mathrm{dl}$, creatinina $1,2 \mathrm{mg} / \mathrm{dl}$, resto (GOT, GPT, LDH, CPK, $\mathrm{Na}^{+}, \mathrm{K}^{+}, \mathrm{Cl}^{-}$) normal.

En consulta de Dermatología se le realizó una biopsia de una de las lesiones de la pared abdominal, con la siguiente descripción macroscópica: fragmento cutáneo que muestra ocupando todos los niveles de la dermis e infiltrando hipodermis una proliferación celular que se extiende en sábana y está compuesta por células de tamaño pequeño-intermedio, de núcleo central ligeramente hendido y citoplasma escaso basófilo. Dichas células muestran positividad para CD4, CD15, CD43 y CD68 siendo negativas para CD20 y CD3. Diagnóstico anatomopatológico: fragmento cutáneo con cambios compatibles con leucemia cutis.

Se reintrodujo el tratamiento con hidroxiurea y las lesiones remitieron a los 3 meses, sin volverse a manifestar nuevamente. A los 16 meses del diagnóstico inicial de LMMC el paciente falleció tras un episodio de hematemesis por hemorragia digestiva alta e insuficiencia renal crónica reagudizada.

\section{COMENTARIOS}

En la literatura actual se carece de datos epidemiológicos con respecto a la incidencia y prevalencia de la $\mathrm{LC}^{3}$. A pesar de que la leucemia linfocítica crónica (LLC) es el tipo de leucemia más frecuente, se ha observado que la LC se manifiesta mayormente en pacientes con leucemia mieloide aguda (LMA).

En un $30-45 \%$ de los pacientes con LC, las lesiones pueden ser previas a la demostración de la enfermedad leucémica en sangre o médula ósea ${ }^{1,2}$.

La morfología clínica de las lesiones por LC no suele ser patognomónicas del tipo de leucemia que afecta al paciente, excepto los cloromas, que se consideran lesiones específicas de LMA, aunque se han documentado también en enfermedades mieloproliferativas ${ }^{3}$. La mucosa oral y las superficies palmo-plantares son las áreas corporales que con menor frecuencia están afectadas.

De acuerdo a la morfología clínica, distribución y localización de las lesiones, deben considerarse diferentes diagnósticos diferenciales, tales como metástasis, linfomas, sarcoma de Kaposi, erupción secundaria a drogas, exantema viral, exantema por sífilis, pitiriasis rosada, vasculitis, epidermolisis bullosa autoinmune, eritrodermia idiopática, úlceras idiopáticas, lupus eritematoso y fibromatosis gingival idiopática ${ }^{1}$.

En un estudio de 15 pacientes con LMA y LC se vio que tras la implementación de quimioterapia remitieron los signos clínicos de LC, sin que se observaran recurrencias en el $75 \%$ de los pacientes, de los cuales, el $90 \%$ fallecieron al 
cabo de 12 meses por causas relacionadas con la leucemia ${ }^{4}$. Con lo cual, no debe asumirse la respuesta al tratamiento quimioterapéutico como indicador pronóstico del curso de la enfermedad.

La LC debe ser considerada, pues, como una manifestación sistémica de mal pronóstico, en la que la mayoría de los pacientes tendrán una esperanza de vida menor de un año, con un promedio de sobrevida de 1,3-3,6 meses posterior a su diagnóstico ${ }^{5}$.

\section{BIBLIOGRAFÍA}

1. Wagner G, Fenchel K, Back W, Schulz A, Sachse MM. Leukemia cutis - epidemiology, clinical presentation,

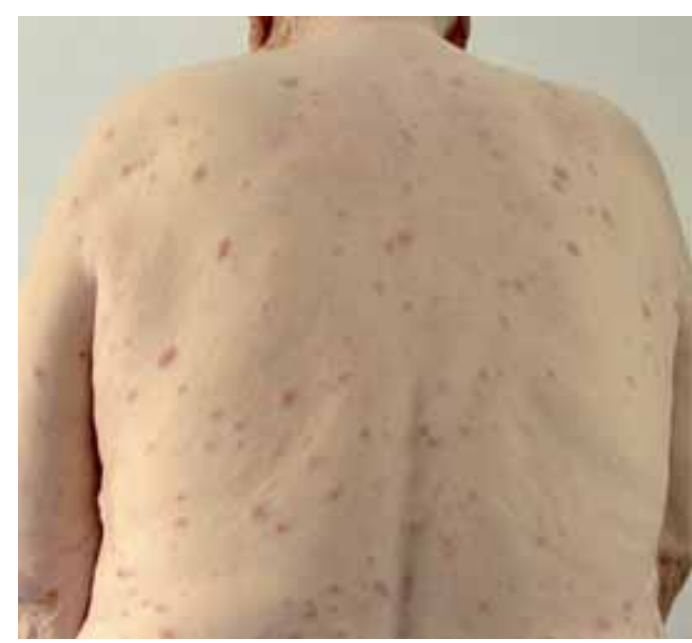

Figura 1. Lesiones en tronco and differential diagnoses. J Dtsch Dermatol Ges. 2012;10(1):27-36.

2. Stawiski MA. Skin manifestations of leukemias and lymphomas. Cutis. 1978;21(6):814-8.

3. Mathew RA, Bennett JM, Liu JJ, Komrokji RS, Lancet JE, Naghashpour $\mathrm{M}$ et al. Cutaneous manifestations in CMML: Indication of disease acceleration or transformation to AML and review of the literature. Leuk Res. 2012;36(1):72-80.

4. Agis $\mathrm{H}$, Weltermann $\mathrm{A}$, Fonatsch $\mathrm{C}$, Haas $\mathrm{O}$, Mitterbauer $G$, Müllauer $L$ et al. A comparative study on demographic, hematological, and cytogenetic findings and prognosis in acute myeloid leukemia with and without leukemia cutis. Ann Hematol. 2002;81(2):90-5.

5. Kaddu S, Zenahlik P, Beham-Schmid C, Kerl H, Cerroni L. Specific cutaneous infiltrates in patients with myelogeneous leukemia: a clinicopathologic study of 26 patients with assessment of diagnostic criteria. J Am Acad Dermatol. 1999;40(6 Pt 1):966-78.

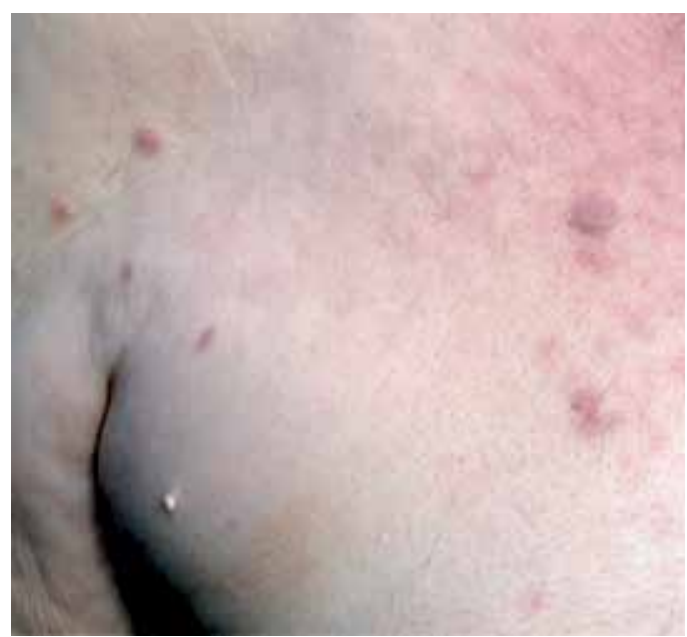

Figura 2. Acercamiento a lesiones ubicadas en cara anterior de torax

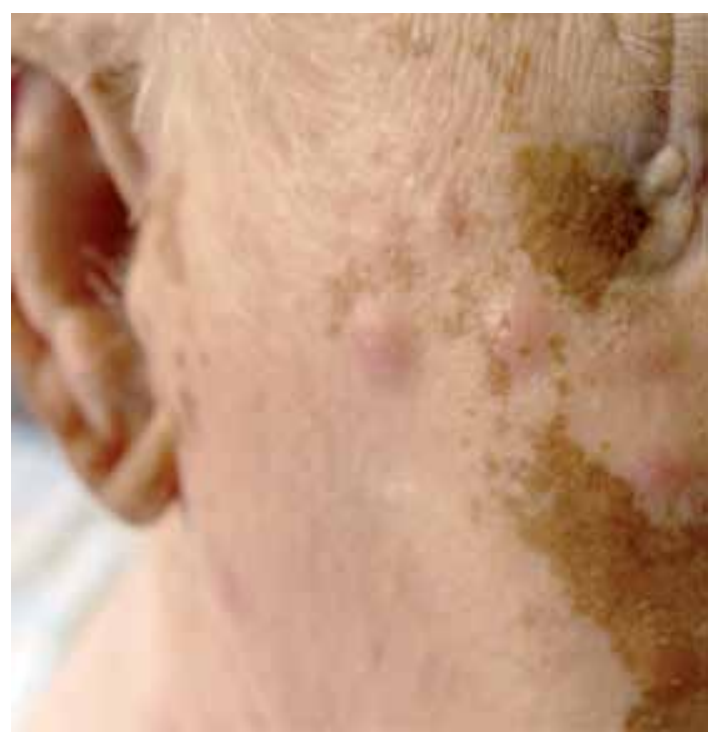

Figura 3. Acercamiento a lesiones en cara donde se aprecian las características nodulares de las mismas. 